\title{
Vulnerability of Ebola Virus outbreak in Bangladesh
}

\begin{abstract}
ASM Alamgir
National Professional Officer (NPO), Pandemic Influenza Surveillance \& Response (PISR) and Laboratory Strengthening CSR/DSE, WHO Country Office, Dhaka, Bangladesh; Email: alamgira@searo.who.int; Cell No.: $+8801715120862$
\end{abstract}

World Health Organization (WHO) has declared Ebola virus infection as a medical emergency ${ }^{1}$. It is also known as Ebola virus disease (EVD) which is formerly known as Ebola haemorrhagic fever (EHF). It is a deadly serious disease; furthermore the case fatality rate is up to $90 \%{ }^{1}$. WHO continues to monitor the evolution of the Ebola virus disease outbreak in Guinea, Liberia, and Sierra Leone. On 30 September 2014, the Pan American Health Organization/World Health Organization (PAHO/WHO) was informed of the first confirmed imported case of EVD in the United States who is an adult with recent travel history to West Africa who developed symptoms compatible with Ebola on 24 September 2014, approximately 4 days after arriving in the United States on 20 September 2014. The patient did not have symptoms when leaving West Africa. The case sought medical care on 26 September 2014 and was admitted into isolation on 28 September 2014 at Texas Health Presbyterian Hospital in Dallas. Samples were sent for testing to the US Center for Disease Control and Prevention in Atlanta, Georgia and at the Texas state laboratory. Results were positive for Ebola virus. Identification of close contacts for further daily monitoring for 21 days after exposure is under way. Given that the case did not exhibit symptoms of Ebola during the flights from
West Africa, contact tracing of people on the same commercial airline flights is not indicated.

Outbreak at USA indicates the seriousness of this disease. Currently it is one of the world's most alarming diseases. During routine surveillance the standard case definition is recommended by WHO-AFRO for the notification of Ebola. The Suspected Ebola for routine surveillance is defined as illness with onset of fever and no response to treatment for usual causes of fever in the area, and at least one of the signs of bloody diarrhoea, bleeding from gums, purpura, bleeding into eyes and urine $^{1}$. The confirmed Ebola case for routine surveillance is defined as a suspected case with laboratory confirmation with positive IgM antibody, positive PCR or viral isolation ${ }^{1}$. During an Ebola outbreak the case definition used by mobile teams or health stations and health centres is different. The suspected case is any person, alive or dead, suffering or having suffered from a sudden onset of high fever and having had contact with a suspected, probable or confirmed Ebola or Marburg case; a dead or sick animal (for Ebola); a mine (for Marburg); OR any person with sudden onset of high fever and at least three of the following symptoms like headaches, vomiting, anorexia / loss of appetite, diarrhea, lethargy, stomach pain, aching muscles or joints, difficulty swallowing, 
breathing difficulties, hiccup OR any person with inexplicable bleeding OR any sudden, inexplicable death ${ }^{1}$. During case definition for exclusive use by hospitals and surveillance teams the probable case is defined as any suspected case evaluated by a clinician OR any deceased suspected case (where it has not been possible to collect specimens for laboratory confirmation) having an epidemiological link with a confirmed case. Again, laboratory confirmed case is defined as any suspected or probably cases with a positive laboratory result. Laboratory confirmed cases must test positive for the virus antigen, either by detection of virus RNA by reverse transcriptase-polymerase chain reaction (RT- PCR), or by detection of IgM antibodies directed against Marburg or Ebola $^{1}$. On the other hand non-case is defined as any suspected or probable case with a negative laboratory result with no specific antibodies, RNA or specific detectable antigens ${ }^{1}$.

The infection is transmitted by direct contact with the blood, body fluids and tissues of infected animals or people ${ }^{2}$. When an infection does occur in humans, there are several ways in which the virus can be transmitted to others like direct contact with the blood or secretions of an infected person and exposure to objects. The viruses that cause Ebola HF are often spread through families and friends because they come in close contact with infectious secretions when caring for ill persons. Health-care workers have frequently been infected while treating patients with suspected or confirmed $\mathrm{EVD}^{2}$. This has occurred through close contact with patients when infection control precautions are not strictly practiced. It has been established that the resource constraints country like
Bangladesh has less aware about the self precaution to avoid infection. Severely ill patients require intensive supportive care. Regarding this issue WHO and Ministry of Health \& Family Welfare of Bangladesh have taken the initiative to establish a separate ICU supported hospital for the management of Ebola virus infected patients. Ebola virus disease outbreaks can devastate families and communities; however, the infection can be controlled through the use of recommended protective measures in clinics and hospitals, at community gatherings, or at home ${ }^{2}$. In addition to that environments contaminated with such fluids are also responsible for transmission of EV. Furthermore, during burial ceremonies mourners have direct contact with the body of the deceased person and this can also play a role in the transmission of Ebola virus. It is interesting that men who have recovered from the disease can still transmit the virus through their semen for up to 7 weeks after recovery from illness. Therefore, apparently cure patient is still very dangerous for the transmission of EV.

Cited as: Alamgir ASM. Vulnerability of Ebola Virus outbreak in Bangladesh. Bangladesh $\mathbf{J}$ Infect Dis, 2014;1(1):1-2]

\section{References}

1. WHO. Case definition recommendations for Ebola or Marburg Virus Diseases; 2012; viewed on: 18 September 2014; Web address: http:/www.afro.who.int/en/clusters-a-programmes/dpc/integrateddiseasesurveillance/features 2775 -technical-guidelines-for-integrateddisease-surveillance-and-response-inthe-african-region.html]

2. Elisha A, Adegboro B. Ebola Virus Diseases. African Journal of Clinical and Experimental Microbiology; 15:117-121 